\title{
Momentum relaxation of a mobile impurity in a one-dimensional quantum gas
}

\author{
E. Burovski, ${ }^{1}$ V. Cheianov, ${ }^{1}$ O. Gamayun, ${ }^{1,2}$ and O. Lychkovskiy ${ }^{1,3,4}$ \\ ${ }^{1}$ Physics Department, Lancaster University, Lancaster LAI 4YB, United Kingdom \\ ${ }^{2}$ Bogolyubov Institute for Theoretical Physics, 14-b Metrolohichna str., Kyiv 03680, Ukraine \\ ${ }^{3}$ Institute for Theoretical and Experimental Physics, 25 B. Cheremushkinskaya str., Moscow 117218, Russia \\ ${ }^{4}$ Russian Quantum Center, Novaya St. 100A, Skolkovo, Moscow Region 143025, Russia
}

(Received 6 September 2013; revised manuscript received 12 November 2013; published 14 April 2014)

\begin{abstract}
We investigate the time evolution of the momentum of an impurity atom injected into a degenerate TonksGirardeau gas. We establish that given an initial momentum $p_{0}$ the impurity relaxes to a steady state with a nonvanishing momentum $p_{\infty}$. The nature of the steady state is found to depend drastically on whether the masses of the impurity and the host are equal. This is due to multiple coherent scattering processes leading to a resonant interaction between the impurity and the host in the case of equal masses. The dependence of $p_{\infty}$ on $p_{0}$ remains nontrivial even in the limit of vanishing interaction between the impurity and host particles. In this limit $p_{\infty}\left(p_{0}\right)$ is found explicitly.
\end{abstract}

DOI: 10.1103/PhysRevA.89.041601

PACS number(s): 03.75.Ss, 05.30.Fk

Introduction. Laws governing the motion of a mobile particle through a fluid provide a powerful insight into the fluid's dynamical properties. For this reason impurities immersed in quantum fluids such as superfluid ${ }^{4} \mathrm{He}$ and Fermi liquid ${ }^{3} \mathrm{He}$ have been a continued subject of study since late 1950s [1]. A remarkable class of quantum fluids, which are neither superfluids nor Fermi liquids, is found in one spatial dimension (1D) [2]. Interest in impurities moving through such fluids started with works on the Fermi edge singularity in inorganic quantum wires [3-5] and the mobility of a heavy particle in a Luttinger liquid [6]. Several distinctive features of the motion of an impurity in 1D have been predicted theoretically such as weak violation of superfluidity [7], non-Markovian relaxation patterns rooted in power-law singularities of the fluid's spectral function [8], logarithmic subdiffusion [9], quantum flutter [10,11], and quasi-Bloch momentum oscillations [12].

Early studies, both theoretical and experimental, were mainly concerned with equilibrium spectral characteristics and linear response properties of an impurity. At present, however, the focus is shifting towards the analysis of dynamic, far-fromequilibrium phenomena [13]. This is fuelled by tremendous experimental progress achieved over the past decade in the area of ultracold atomic gases. First elongated traps containing interacting 1D Bose gases were demonstrated 10 years ago [14-16]. By 2009 sufficient control over the system became available to conduct experiments with an ensemble of impurity atoms under a constant drag force [17] and out-of-equilibrium impurity clouds injected into the host [18]. Furthermore, development of single-atom-resolved control [19] and imaging techniques [20,21] opens unprecedented experimental opportunities such as a direct observation of the motion of an individual impurity atom in a one-dimensional gas [22].

On the theory side, several complementary approaches are being developed. For bosonic hosts in the Bogoliubov limit remarkable results have been obtained by methods of quantum hydrodynamics $[12,23,24]$. In particular, it was predicted that the momentum of an impurity driven by a constant force may exhibit oscillations resembling the Bloch oscillations in an ideal crystal [12] (however, this prediction was criticized in Ref. [25]).
A Bethe-ansatz solvable model of an impurity injected in a Tonks-Girardeau (TG) host has been considered in Ref. [10]. Using numerical summation of form-factor series for a finite-size system Ref. [10] investigated an impurity's momentum relaxation at intermediate time scales. The momentum of the impurity as a function of time was found to follow a counterintuitive pattern resembling underdamped periodic oscillations around some nonzero average. This phenomenon was dubbed the "quantum flutter" [10]. Subsequent numerical simulations based on matrix product states extended the results of Ref. [10] to a nonintegrable case [11]. The results of Refs. $[10,11]$ suggest, in particular, the possibility of a nonvanishing steady-state momentum of the impurity. Considering the absence of superfluidity in one dimension [7], such incomplete momentum relaxation contradicts equipartition of energy and signals the failure of thermalization in the system. The purpose of the present Rapid Communication is to explain the physical mechanism responsible for this phenomenon and to develop a complete analytical theory of the formation of the steady state in a certain perturbative limit.

To this end, we investigate the relaxation of the momentum of an impurity weakly interacting with a degenerate $(T=0)$ Tonks-Girardeau gas [26], focusing on the infinite time steady state of the system. Within the Boltzmann kinetic theory we find the dependence of the infinite-time momentum of the impurity $p_{\infty}$ on the initial momentum $p_{0}$ and explain the mechanism by which $p_{\infty}$ is nonzero. We find that when the masses of the impurity and the host particle are equal, the Boltzmann theory breaks down. In this case, we resort to an alternative approach based on the Bethe ansatz solution for a pointlike interaction. We first develop a method of dealing with form factor expansions numerically. Using the insight from numerical simulations, we perform a controllable asymptotic analysis of the problem to obtain a closed-form expression for $p_{\infty}\left(p_{0}\right)$.

Problem formulation. We consider a single mobile impurity of mass $m_{i}$ immersed in the TG gas of particles of mass $m_{h}$. In the following we exploit the exact spectral equivalence between the TG gas and a gas of noninteracting Fermi particles [26] and refer to the host particles as "fermions". We assume a short-range repulsive interaction between the impurity and the host fermions such that the total Hamiltonian 
is

$$
\hat{H}=\hat{H}_{h}^{0}+\hat{H}_{i}^{0}+\gamma \frac{k_{F}}{\pi m_{h}} \int d x \hat{\rho}_{h}(x) \hat{\rho}_{i}(x) .
$$

Here $\hat{H}_{h}^{0}, \hat{H}_{i}^{0}$ and $\hat{\rho}_{h}(x), \hat{\rho}_{i}(x)$ are the Hamiltonians and density operators of the host fermions and the impurity, respectively, and $\gamma$ is the dimensionless constant. Depending on the mass ratio $\eta \equiv m_{i} / m_{h}$, we distinguish between the cases of "light impurity", $\eta<1$, and "heavy impurity", $\eta>1$. We define the Fermi momentum $k_{F} \equiv \pi \rho$, where $\rho$ is the host particle density.

We are interested in the time evolution of the system from an initial state being a direct product of the ground state of $\hat{H}_{h}^{0}$ (which is merely a Fermi sea of the host particles) and a plane-wave state of the impurity with momentum $p_{0}>0$. Our main goal is to find the impurity momentum distribution function $w_{p}(t)$, investigate its $t \rightarrow \infty$ limit, which we denote by $w_{p_{0} \rightarrow p}^{\infty}$, and calculate the infinite-time momentum $p_{\infty}=\sum_{p} p w_{p_{0} \rightarrow p}^{\infty}$.

Kinematics. We begin our analysis from semiclassical considerations. Kinematics of a two-particle scattering in 1D is completely characterized by two momenta, e.g., the initial, $q$, and final, $k$, momenta of the impurity. Given these, the initial and final momenta of the host particle are completely fixed by two conservation laws. In addition, the Pauli principle restricts possible values of $k$ and $q$ to a certain region in the $(q, k)$ plane. This kinematically allowed region is shown on Fig. 1. We see that the allowed region exists for all
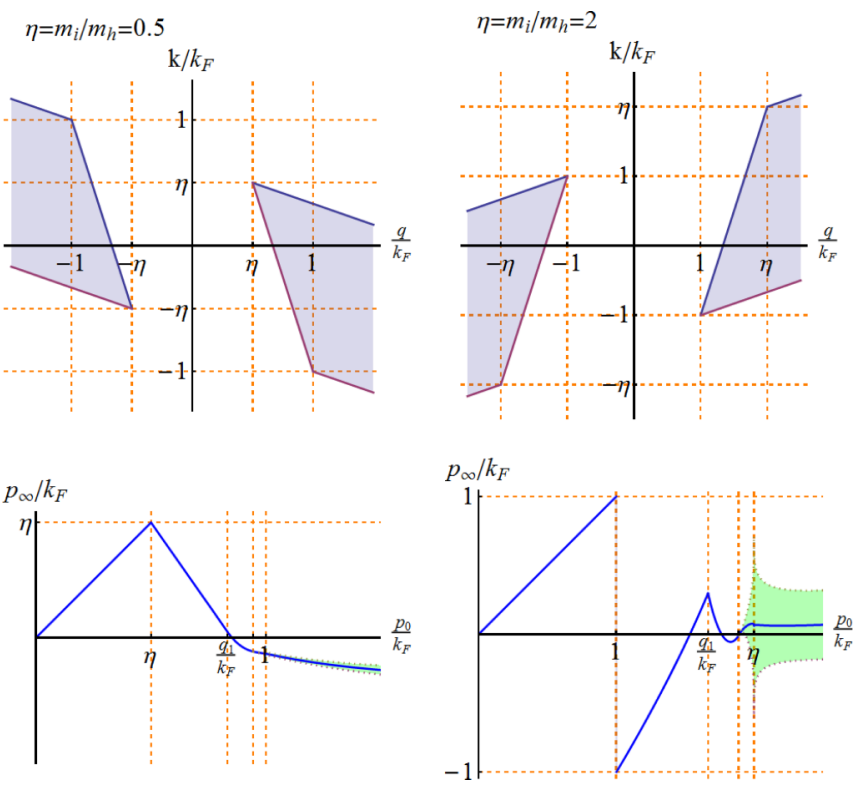

FIG. 1. (Color online) Upper panels: Kinematically allowed regions for a single pairwise scattering in the cases of the light (left) and heavy (right) impurity. The final momentum of the impurity, $k$, is shown vs the initial momentum of the impurity, $q$. Lower panels: The impurity momentum at infinite time, $p_{\infty}$, as a function of the initial momentum, $p_{0}$, for the light (left) and heavy (right) impurity. Solid blue line shows an iterative solution (two iterations) of Eq. (5). Shaded area (green online) represents the maximum error: The exact solution of Eq. (5) lies inside this area. Notice a much better convergence of iterations in the case of light impurity. $|q|>q_{0}$, where $q_{0} \equiv k_{F} \min \{1, \eta\}$, and its boundaries are piecewise linear functions of $q$. We denote these functions by $u(q)$ for the upper boundary and $d(q)$ for the lower one.

If the initial momentum of the impurity satisfies $\left|p_{0}\right|<q_{0}$, Pauli blocking precludes any scattering, and the impurity's momentum is conserved. For $\left|p_{0}\right|>q_{0}$, scattering events continue until the impurity momentum drops below $q_{0}$, after which scattering stops. There exists a momentum $q_{1}>q_{0}$ such that, whenever $q_{0}<\left|p_{0}\right|<q_{1}$, the impurity momentum drops below $q_{0}$ in a single scattering event. Furthermore, there exists an infinite ascending sequence $\left\{q_{n}\right\}$ such that $\left|p_{0}\right|<q_{n}$ implies that the impurity momentum drops below $q_{0}$ in no more than $n$ collisions. The recursive definition for the sequence reads $q_{n-1}=\max \left\{\left|u\left(q_{n}\right)\right|,\left|d\left(q_{n}\right)\right|\right\}$; the sequence converges to $q_{\infty}=k_{F} \max \{1, \eta\}$ with $n \rightarrow \infty$. Note that the case of equal masses $(\eta=1)$ is special: The whole sequence $\left\{q_{n}\right\}$ collapses to a single point, $q_{n}=k_{F}$.

Now consider the classical evolution of the momentum distribution function of an impurity. If $\left|p_{0}\right|<q_{n}$, no more than $n$ collisions bring the system to a steady state in which the impurity's momentum distribution function $w_{p_{0} \rightarrow p}^{\infty}$ has a finite support $p \in\left[-q_{0}, q_{0}\right]$. There are no symmetries of the problem to prevent this function from having a nonvanishing first moment. Therefore, in general, $p_{\infty} \neq 0$. This conclusion is supported by a nonperturbative fully quantum treatment: When $p_{0}$ lies in a certain range, one can rigorously prove that $p_{\infty}$ is nonzero [27].

Applicability of the Boltzmann equation. The above kinematical considerations are a good starting point for the application of Boltzmann's kinetic theory. The latter requires the validity of Fermi golden rule, which is ensured by the smallness of the dimensionless coupling constant, $\gamma \ll 1$, and by a narrow impurity level spacing (as compared to the collision rate) which implies $\gamma^{2} N \gg 1$ for a system of $N$ particles. Apart from these two constraints, the dimensionality of the problem imposes extra conditions. Indeed, the validity of Boltzmann's equation relies on the Lorentzian shape of particle's spectral function such as in Fermi liquid theory [28]. Generally, in 1D systems, spectral functions of particles exhibit essentially non-Lorentzian shapes in the vicinity of the mass shell [2,8,29] (an exception from this rule was discussed in Ref. [30]). The source of this peculiarity is virtual longwavelength modes which produce a logarithmically divergent contribution to the self-energy, $\delta \Sigma \sim \gamma^{2} \ln N$. In order for Boltzmann's equation to work one needs to suppress the divergence and impose $\gamma^{2} \ln N \ll 1$. Finally, another divergent contribution to self-energy arises from the ladder diagrams in the equal mass limit $m_{i} \rightarrow m_{h}$. The physical meaning of this divergence will be explained below. The requirement that the ladder corrections can be neglected results in the condition $|\eta-1| \gg \gamma$.

Analysis of the Boltzmann equation. The kinetic equation reads as follows [31]:

$$
\begin{gathered}
\dot{w}_{k}(t)=-\Gamma_{k} w_{k}(t)+\sum_{q} \Gamma_{q \rightarrow k} w_{q}(t), \\
\Gamma_{q \rightarrow k}=\frac{\gamma^{2}}{\pi^{2}} \frac{k_{F}^{2}}{L m_{h}} \frac{\theta(d(q)<k<u(q))}{|q-k|} .
\end{gathered}
$$


Here $\Gamma_{q \rightarrow k}$ is the partial width and $\Gamma_{k} \equiv \sum_{q} \Gamma_{k \rightarrow q}$ is the total width. Due to kinematical constraints reflected in step functions in Eq. (3), the kinetic equation (2) leads to the following integral equation on the asymptotic distribution:

$w_{p_{0} \rightarrow k}^{\infty}=\theta\left(q_{0}-|k|\right)\left(\mathcal{P}_{p_{0} \rightarrow k}^{(1)}+\sum_{q \in \mathcal{R}\left(p_{0}\right)} \mathcal{P}_{p_{0} \rightarrow q}^{(1)} w_{q \rightarrow k}^{\infty}\right)$,

where $\mathcal{P}_{p_{0} \rightarrow k}^{(1)}=\Gamma_{p_{0} \rightarrow k} / \Gamma_{p_{0}}$ is the probability that the impurity changes its momentum from $p_{0}$ to $k$ in a single scattering event, and $\mathcal{R}\left(p_{0}\right) \equiv\left[d\left(p_{0}\right), u\left(p_{0}\right)\right] \backslash\left[-q_{0}, q_{0}\right]$ is a kinematically determined integration region. Calculating the first moment of the distribution (4) (with respect to $k$ ) we find the integral equation for the asymptotic momentum,

$$
\begin{aligned}
& p_{\infty}\left(p_{0}\right)=p_{\infty}^{(1)}\left(p_{0}\right)+\sum_{q \in \mathcal{R}\left(p_{0}\right)} \mathcal{P}_{p_{0} \rightarrow q}^{(1)} p_{\infty}(q), \\
& p_{\infty}^{(1)}\left(p_{0}\right) \equiv \sum_{k=-q_{0}}^{q_{0}} k \mathcal{P}_{p_{0} \rightarrow k}^{(1)} .
\end{aligned}
$$

For $\left|p_{0}\right|<q_{0}$ the momentum does not relax, $p_{\infty}\left(p_{0}\right)=p_{0}$. The coupling strength $\gamma$ cancels out from Eqs. (4) and (5), and in Eq. (2) it can be absorbed in rescaling of time.

Equations (2), (4), and (5) can be solved by iterations. In particular, the first iteration for asymptotic momentum is $p_{\infty}^{(1)}\left(p_{0}\right)$. The $n$-th iteration takes into account classical evolution paths which involve no more than $n$ scattering events. If $q_{n-1}<\left|p_{0}\right|<q_{n}$, then $n$ iterations lead to an exact solution. Fewer iterations give an approximate solution. If $\left|p_{0}\right|>q_{\infty}$, any finite number of iterations gives an approximate solution. The convergence of iterations is well controlled. The error at the $n$-th step of the iterative solution of Eq. (5) is bounded from above by $q_{0}$ times the probability to scatter below $q_{0}$ in more than $n$ collisions. The solution of Eq. (5) is plotted in Fig. 1 . Note that since $p_{\infty} \in\left[-q_{0}, q_{0}\right]$, the asymptotic velocity of an infinitely heavy impurity vanishes, which is consistent with Refs. [7,32].

The solution $p_{\infty}\left(p_{0}\right)$ of Eq. (5) has nonanalyticities at $p_{0}=$ $q_{n}, n=0,1, \ldots, \infty$. The most prominent one is a jump which occurs at $p_{0}=k_{F}$ when $m_{i}>m_{h}$; see Fig. 1 . In the vicinity of $k_{F}$ we find

$$
p_{\infty}\left(p_{0}\right)=\left\{\begin{array}{ll}
p_{0}, & p_{0}<k_{F} \\
-k_{F}+\frac{m_{i}^{2}+m_{h}^{2}}{m_{i}^{2}-m_{h}^{2}}\left(p_{0}-k_{F}\right), & p_{0}>k_{F}
\end{array},\right.
$$

Other type of singularity is a kink at $p_{0}=k_{F} \eta$, where the velocity of the impurity equals the Fermi velocity, resulting in a forward scattering anomaly. We expect these singularities to be smoothed out by quantum corrections in higher orders in $\gamma$. Note that taking the $m_{i} \rightarrow m_{h}$ limit is nontrivial and is discussed below.

Equal masses. In this case, kinematics of a two-body collision reduces to an exchange of momenta. Thus, the very first scattering event brings the impurity to the state with $k \in\left[-k_{F}, k_{F}\right]$ and creates a hole with momentum $-k$. From this moment on, the impurity and a hole move with the same velocity. Further multiple coherent scatterings in this two-body system allow the momentum of the impurity to migrate unrestricted in the range $\left[-k_{F}, k_{F}\right]$. Thus one might expect that $p_{\infty}=0$. Below we demonstrate that this intuitive expectation fails.

The Boltzmann equation does not capture multiple coherent scattering processes. However, the contact interaction Hamiltonian (1) with $m_{i}=m_{h}$ is Bethe ansatz integrable $[5,33,34]$, which allows us to calculate $p_{\infty}$ explicitly in the same limit, $\gamma \rightarrow 0, \gamma^{2} N \rightarrow \infty, \gamma^{2} \ln N \rightarrow 0$, as for Boltzmann's theory above [35].

Bethe ansatz and form-factor expansion. For a finite number of particles of the background gas, $N$, eigenstates of the Hamiltonian (1), $\left|\psi_{\lambda}\right\rangle$, are labeled by ordered sets $\lambda=\left\{n_{0}, n_{1}, \ldots, n_{N}\right\}$ of $N+1$ distinct integers. The value of the asymptotic momentum follows from the form-factor expansion,

$$
\left.p_{\infty}=\sum_{\lambda}\left\langle\psi_{\lambda}\left|\widehat{P}_{i}\right| \psi_{\lambda}\right\rangle \mid\left\langle\psi_{\lambda}\right| \text { in }\right\rangle\left.\right|^{2}
$$

where $\widehat{P}_{i}$ is the impurity momentum operator and $\mid$ in $\rangle$ is the initial state of the system, and the summation is over the infinite complete set of eigenstates. Explicit determinant representations for the matrix elements entering (7) have been found in Ref. [10]. However, evaluation of the sum over intermediate states remains a challenge. A general analytical solution is unknown, and numerically the difficulty is to find an efficient and controllable way of selecting most relevant contributions to the Eq. (7). While rather sophisticated ways of scanning the Hilbert space have been developed [10,36], the task remains difficult. We have been able to both significantly advance numerical technique and develop successful analytic approach in the perturbative limit.

Bethe ansatz and numerics. We note that the structure of Eq. (7) naturally lends itself to a stochastic sampling of the Hilber space: Instead of evaluating the sum in Eq. (7) in a predetermined order, we construct a random walk in the space of ordered sets $\lambda$, based on the Metropolis algorithm [37] with transition probabilities proportional to $\mid\left\langle\psi_{\lambda}\right|$ in $\rangle\left.\right|^{2}$. This way, the algorithm automatically finds the most relevant regions of the Hilbert space. In practice, we only use local updates of the configurations (i.e., at each step of the Markov process we only change one or two integers in the ordered set $\lambda$ ) and observe a very quick convergence of the sum (7). Detailed description of the algorithm will be given elsewhere [38].

We do numerics on systems with up to $405+1$ particles, which is an order of magnitude improvement compared to earlier approaches $[10,11]$. We find a substantial dependence of $p_{\infty}$ on $N$, which persists up to the largest available $N$; see Fig. 2. Thus, a thorough investigation of finite-size corrections is essential for extracting the thermodynamic limit behavior. We reserve such analysis for a separate publication [38].

Concentrating on the regime of moderate $\gamma$, we find that Eq. (7) is dominated by the one-parameter family of states $\mathfrak{s}=\{\lambda(\tilde{n}), \tilde{n}>N / 2\}$, where $\lambda(\tilde{n}) \equiv$ $\{-(N-1) / 2,-(N-3) / 2, \ldots,(N-1) / 2, \tilde{n}\}$; see Fig. 2 for an illustrative example. In fact, in the limit $\gamma \rightarrow 0$ the family $\mathfrak{s}$ can be investigated analytically.

Bethe ansatz and asymptotic analysis. In the limit $\gamma^{2} \ln N \rightarrow 0$ and $\gamma^{2} N \rightarrow \infty$ we are able to obtain an explicit asymptotic expressions for the form factors $\left\langle\psi_{\lambda}\right|$ in $\rangle$ and $\left\langle\psi_{\lambda}\left|\hat{P}_{i}\right| \psi_{\lambda}\right\rangle$ for $\lambda \in \mathfrak{s}$. Furthermore, we prove that the states 


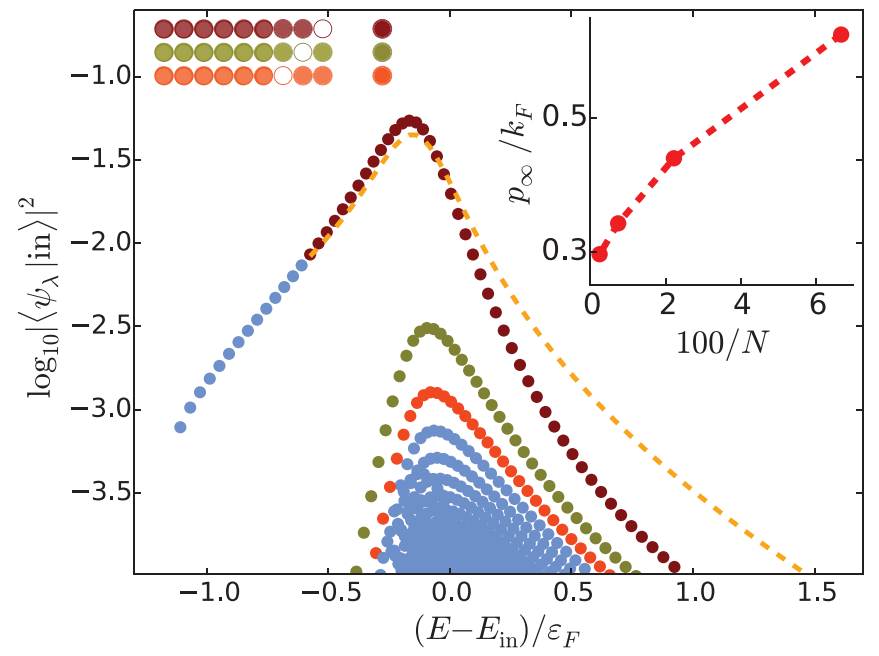

FIG. 2. (Color online) Overlaps $\mid\left\langle\psi_{\lambda}\right|$ in $\rangle\left.\right|^{2}$ versus energy $E$ (relative to the in-state energy $E_{\text {in }}$ ) for $N=135, L=405, \gamma=3$, and $p_{0}=1.2 k_{F}$. Several families of states are clearly visible. The diagram in the upper left corner shows schematically the structure of sets $\lambda$ for the top three families, including the dominant family $\mathfrak{s}$ (maroon). The dashed curve corresponds to the asymptotic expression obtained in the limit $\gamma^{2} \ln N \rightarrow 0, \gamma^{2} N \rightarrow \infty$. Inset demonstrates the finite-size dependence of $p_{\infty}$ at $p_{0}=1.6 k_{F}$ and $\gamma=3$.

from $\mathfrak{s}$ saturate the sum rule, $\sum_{\lambda \in \mathfrak{s}} \mid\left\langle\psi_{\lambda}\right|$ in $\rangle\left.\right|^{2}=1$. This way, Eq. (7) yields [38]

$$
p_{\infty}=p_{0}-\theta\left(\left|p_{0}\right|-k_{F}\right) \frac{p_{0}^{2}-k_{F}^{2}}{2 k_{F}} \ln \frac{p_{0}+k_{F}}{p_{0}-k_{F}} .
$$

Discussion and outlook. It is interesting now to compare Eq. (8) with an exact solution of Eq. (5) in the case of equal masses,

$$
p_{\infty}^{B}=p_{0}-2 k_{F} \theta\left(\left|p_{0}\right|-k_{F}\right)\left(\ln \frac{p_{0}+k_{F}}{p_{0}-k_{F}}\right)^{-1} .
$$

We see that the Boltzmann theory fails to produce a correct result at $m_{i}=m_{h}$, which is the consequence of the resonant interaction discussed earlier. In the vicinity of the $\gamma=0, \eta=1$ point in the $(\gamma, \eta)$ plane the validity of Eqs. (8) and (9) depends on the $|\eta-1| / \gamma$ ratio. Equation (8) is valid for $|\eta-1| / \gamma \ll 1$ and Eq. (9) for $|\eta-1| / \gamma \gg 1$. From a mathematical point of view this means that the limits $\eta \rightarrow 1$ and $\gamma \rightarrow 0$ do not commute. At any finite $\gamma$ there is no discontinuity of $p_{\infty}(\eta)$ at $\eta=1$. This is consistent with findings [10,11].

It is interesting to discuss our results in the context of thermalization [13]. When $\left|p_{0}\right|>q_{0}$ the impurity is kinematically allowed to exchange energy and momentum with the bath (host). If such an exchange had led to a complete thermalization, equipartition would have implied $p_{\infty}=0$. We see, however, that this is not the case no matter whether the model is integrable. This seems to be one of the rare examples of the thermalization failure in a local, nonintegrable model without disorder (see, e.g., the discussion in Ref. [39]).

Finally, we outline directions for further development. Equation (2) can be generalized to describe the motion of the impurity under an external force and at nonzero temperature. This way, one can investigate the asymptotical momentum as a function of force and describe the impurity dynamics in TG gas at an arbitrary ratio $m_{i} / m_{h} \neq 1$ [40]. The case of equal masses, where the Boltzmann equation fails, requires a special treatment. To this end, we developed Bethe ansatz-based tools, which can be extended for studying an integrable system with applied force and/or finite couplings.

Acknowledgments. We thank M. Zvonarev and L. Glazman for illuminating discussions. The present work was supported by ERC Grant No. 279738-NEDFOQ. E.B. acknowledges partial support from Lancaster University via ECSG Grant No. SGS/18/01. O.L. acknowledges partial support via Grant No. RFBR-12-02-00193 and the Leading Scientific Schools Grant No. 3830.2014.2.
[1] I. M. Khalatnikov and V. N. Zharkov, J. Exp. Theor. Phys. 32, 1108 (1957); L. Meyer and F. Reif, Phys. Rev. 110, 279 (1958); N. Prokofev, Int. J. Mod. Phys. B 7, 3327 (1993).

[2] T. Giamarchi, Quantum Physics in One Dimension (Oxford University Press, Oxford, 2003).

[3] J. Calleja, A. Goni, B. Dennis, J. Weiner, A. Pinczuk, S. Schmitt-Rink, L. Pfeiffer, K. West, J. Müller, and A. Ruckenstein, Solid State Commun. 79, 911 (1991).

[4] T. Ogawa, A. Furusaki, and N. Nagaosa, Phys. Rev. Lett. 68, 3638 (1992).

[5] H. Castella and X. Zotos, Phys. Rev. B 47, 16186 (1993).

[6] A. H. Castro Neto and M. P. A. Fisher, Phys. Rev. B 53, 9713 (1996).

[7] G. E. Astrakharchik and L. P. Pitaevskii, Phys. Rev. A 70, 013608 (2004).

[8] A. Imambekov, T. L. Schmidt, and L. I. Glazman, Rev. Mod. Phys. 84, 1253 (2012).
[9] M. B. Zvonarev, V. V. Cheianov, and T. Giamarchi, Phys. Rev. Lett. 99, 240404 (2007).

[10] C. J. Mathy, M. B. Zvonarev, and E. Demler, Nat. Phys. 8, 881 (2012).

[11] M. Knap, C. J. M. Mathy, M. Ganahl, M. B. Zvonarev, and E. Demler, Phys. Rev. Lett. 112, 015302 (2014).

[12] D. M. Gangardt and A. Kamenev, Phys. Rev. Lett. 102, 070402 (2009).

[13] A. Polkovnikov, K. Sengupta, A. Silva, and M. Vengalattore, Rev. Mod. Phys. 83, 863 (2011).

[14] H. Moritz, T. Stöferle, M. Köhl, and T. Esslinger, Phys. Rev. Lett. 91, 250402 (2003).

[15] T. Kinoshita, T. Wenger, and D. Weiss, Science 305, 1125 (2004).

[16] B. Paredes, A. Widera, V. Murg, O. Mandel, S. Fölling, I. Cirac, G. V. Shlyapnikov, T. W. Hänsch, and I. Bloch, Nature 429, 277 (2004). 
[17] S. Palzer, C. Zipkes, C. Sias, and M. Köhl, Phys. Rev. Lett. 103, 150601 (2009).

[18] J. Catani, G. Lamporesi, D. Naik, M. Gring, M. Inguscio, F. Minardi, A. Kantian, and T. Giamarchi, Phys. Rev. A 85, 023623 (2012).

[19] N. Spethmann, F. Kindermann, S. John, C. Weber, D. Meschede, and A. Widera, Phys. Rev. Lett. 109, 235301 (2012).

[20] W. S. Bakr, A. Peng, M. E. Tai, R. Ma, J. Simon, J. I. Gillen, S. Foelling, L. Pollet, and M. Greiner, Science 329, 547 (2010).

[21] J. F. Sherson, C. Weitenberg, M. Endres, M. Cheneau, I. Bloch, and S. Kuhr, Nature 467, 68 (2010).

[22] T. Fukuhara, A. Kantian, M. Endres, M. Cheneau, P. Schauß, S. Hild, D. Bellem, U. Schollwöck, T. Giamarchi, C. Gross, et al., Nat. Phys. 9, 235 (2013).

[23] M. Schecter, A. Kamenev, D. M. Gangardt, and A. Lamacraft, Phys. Rev. Lett. 108, 207001 (2012).

[24] M. Schecter, D. Gangardt, and A. Kamenev, Ann. Phys. 327, 639 (2012).

[25] O. Lychkovskiy, arXiv:1403.7408 (2014).

[26] M. Girardeau, J. Math. Phys. 1, 516 (1960).

[27] O. Lychkovskiy, Phys. Rev. A 89, 033619 (2014).

[28] G. D. Mahan, Many-Particle Physics (Kluwer Academic/ Plenum, New-York, 2000).
[29] M. B. Zvonarev, V. V. Cheianov, and T. Giamarchi, Phys. Rev. B 80, 201102 (2009).

[30] M. Khodas, M. Pustilnik, A. Kamenev, and L. I. Glazman, Phys. Rev. B 76, 155402 (2007).

[31] Rigorous derivation is given in: O. Gamayun, arXiv:1402.7064 (2014).

[32] C. L. Kane and M. P. A. Fisher, Phys. Rev. B 46, 15233 (1992).

[33] J. McGuire, J. Math. Phys. 6, 432 (1965).

[34] D. Edwards, Prog. Theor. Phys. Suppl. 101, 453 (1990).

[35] Note that the breakdown of the Boltzmann kinetic equation in the equal-mass case is not directly related to integrability. In particular, for a finite-range interaction potential the integrability is absent; however, the Boltzmann equation fails for the same kinematical reasons.

[36] J.-S. Caux, J. Math. Phys. 50, 095214 (2009).

[37] N. Metropolis, A. W. Rosenbluth, M. N. Rosenbluth, A. H. Teller, and E. Teller, J. Chem. Phys. 21, 1087 (1953).

[38] E. Burovski, V. Cheianov, O. Gamayun, and O. Lychkovskiy, in preparation. The numerical code we used is available at https://bitbucket.org/burovski/mcba.

[39] C. Gogolin, M. P. Müller, and J. Eisert, Phys. Rev. Lett. 106, 040401 (2011).

[40] O. Gamayun, O. Lychkovskiy, and V. Cheianov, arXiv:1402.6362 (2014). 